\title{
Effect of Elastic Deformation on the Dispersion Characteristics of a Chain of Microcavities
}

\section{Rumyantsev $\mathbf{V}^{1 *}$, Aparajita $\mathbf{U}^{2}$ and Roslyak $\mathrm{O}^{3}$}

${ }^{1}$ A.A. Galkin Donetsk Institute for Physics and Engineering, Donetsk, Ukraine

${ }^{2} B M C C$, CUNY, New York, USA

${ }^{3}$ Fordham University, New York, USA

\begin{abstract}
We study electromagnetic excitations in non-ideal 1D microcavity lattice with the use of the virtual crystal approximation. The effect of elastic deformation on the excitation spectrum of a microcavity chain is numerically modeled for 1D non-ideal microcavity supercrystal containing quantum dots and without them. The adopted approach helps us obtain the dispersion dependence of collective excitation frequencies and the energy. The analytical expressions for polaritonic frequencies, effective mass and group velocities as functions of corresponding quantum dots and vacancies concentrations are obtained.
\end{abstract}

Keywords: 1D Microcavity supercrystal; Electromagnetic excitations; Virtual crystal approximation; Elastic deformation

\section{Introduction}

Concepts developed in physics of crystalline solids, to a large extent, can be applicable to photonic supercrystals. In this connection some promising vistas can be opened up by the so-called polaritonic crystals (PC) [1], which represents a particular type of photonic crystals characterized by a strong coupling between quantum excitations (excitons) and electromagnetic waves. An example of a polaritonic structure is provided by an array of coupled microcavities [2]. Optical modes in microcavity systems have been attracting a considerable attention due to the progress in fabrication of novel optoelectronic devices $[3,4]$. Recently the focus has been on the ability to control the propagation of electromagnetic excitations in the composite structures by modifying their physical properties with external influences (for example, elastic deformation). In this work we make use of some previously developed formalism in photonic structures $[5,6]$ to treat a non-ideal PC formed by a topologically ordered array of coupled microcavities (resonators) containing a system of atomic clusters (quantum dots). Particular attention is paid to the sensitivity of the polaritonic spectrum on the geometry and key parameters of interacting photonic and electronic subsystems.

We study $1 \mathrm{D}$ polaritonic crystal as a topologically ordered system of coupled microcavities (see, for example, [7]) with and without quantum dots. Uniform elastic deformation of 1D structures causes some peculiar effects. Effectively it is possible to achieve the necessary changes of its polaritonic spectrum and other optical properties.

\section{Theoretical Background}

Based on the approach developed in refs. [1,5-8], we consider electromagnetic excitations in a lattice of microcavities composed of $s$ sublattices. Each of the tunnel-coupled microresonators is assumed to possess a single dominating optical mode. Under elastic stress Hamiltonian $\hat{H}(\hat{\varepsilon})$ of resonator-localized electromagnetic excitations is a function of deformation tensor $\hat{\varepsilon}$.

Under assumption that the density of excited states of constituent elements in resonator and atomic systems is a small quantity and within the one-level model and Heitler-London approximation Hamiltonian $\hat{H}(\hat{\varepsilon})$ has the form (ref. [9]):

$$
\begin{aligned}
& \hat{H}(\hat{\varepsilon})=\sum_{\substack{\mathbf{n}, \mathbf{m}, \alpha, \beta, \lambda, \sigma}} D_{\mathbf{n} \alpha, \mathbf{m} \beta}^{\lambda \sigma}(\hat{\varepsilon}) \hat{\Phi}_{\mathbf{n} \alpha \lambda}^{+} \hat{\Phi}_{\mathbf{m} \beta \sigma}=\sum_{\alpha, \beta, \lambda, \sigma} D_{\alpha \beta}^{\lambda \sigma}(\mathbf{k}, \hat{\varepsilon}) \hat{\Phi}_{\alpha \hat{k}}^{+}(\mathbf{k}) \hat{\Phi}_{\beta \sigma}(\mathbf{k}), \\
& D_{\mathbf{n} \alpha, \mathbf{m} \beta}^{11}(\hat{\varepsilon})=\hbar \omega_{\mathbf{n} \alpha}^{a t} \delta_{\mathbf{n} \alpha, \mathbf{m} \beta}+V_{\mathbf{n} \alpha, \mathbf{m} \beta}(\hat{\varepsilon}), D_{\mathbf{n} \alpha, \mathbf{m} \beta}^{22}=\hbar \omega_{\mathbf{n} \alpha}^{p h} \delta_{\mathbf{n} \alpha, \mathbf{m} \beta}-A_{\mathbf{n} \alpha, \mathbf{m} \beta}(\hat{\varepsilon}), \\
& D_{\mathbf{n} \alpha, \mathbf{m} \beta}^{12}(\hat{\varepsilon})=D_{\mathbf{n} \alpha, \mathbf{m} \beta}^{21}(\hat{\varepsilon})=g_{\mathbf{n} \alpha}(\hat{\varepsilon}) \delta_{\mathbf{n} \alpha, \mathbf{m} \beta}, \hat{\Phi}_{\mathbf{n} \alpha}^{\lambda=2}=\hat{\Psi}_{\mathbf{n} \alpha}, \hat{\Phi}_{\mathbf{n} \alpha}^{\lambda=1}=\hat{B}_{\mathbf{n} \alpha}
\end{aligned}
$$

Where $h \omega_{\mathbf{n} \alpha}^{p h}\left(h \omega_{\mathbf{n} \alpha}^{a t}\right)$ is the energy of the photonic mode without (with) a quantum dot localized at $\mathbf{n} \alpha$-th lattice site (resonator); $\hat{\Psi}_{n \alpha}\left(\hat{B}_{\mathrm{n} \alpha}\right)$ is a Bose annihilation operator of the corresponding mode in nodal representation; $A_{\mathrm{n} \alpha \mathbf{m} \beta}(\hat{\varepsilon})$ is the matrix of resonance interaction, which describes an overlap between optical fields of resonators in the $\mathbf{n} \alpha$-th and $\mathbf{m} \beta$-th lattice sites and hence defines the jump probability of the corresponding electromagnetic excitation; $V_{\mathbf{n} \alpha \mathbf{m} \beta}(\hat{\varepsilon})$ is the matrix of resonance interaction between quantum dots embedded in the $\mathbf{n} \alpha$-th and $\mathbf{m} \beta$-th lattice sites; $g_{\mathbf{n} \alpha}(\hat{\varepsilon})$ is the matrix of resonance interaction between quantum dot in the $\lambda, \sigma$-th lattice site with electromagnetic field localized at the same site. In the adopted formalism values 1 and 2 of indices $\lambda, \sigma$ indicate, correspondingly, presence or absence of quantum dots in respective cavities.

Translation invariance of the system under uniform deformations allows for the quasi-crystal representation (summation over $\mathbf{k}$ ) of the matrices $D_{\alpha \beta}^{\lambda \sigma}(\mathbf{k}, \hat{\varepsilon})$ and $\Phi_{\alpha \lambda}(\mathbf{k})$ in the form:

$$
\begin{aligned}
& D_{\alpha \beta}^{\lambda \sigma}(\mathbf{k}, \hat{\varepsilon})=\sum_{\mathbf{m}-\mathbf{n}>0} D_{\mathbf{n} \alpha \mathbf{m} \beta}^{\lambda \sigma}(\hat{\varepsilon}) \exp \left[i \mathbf{k} \cdot\left(\mathbf{r}_{\mathbf{n} \alpha}-\mathbf{r}_{\mathbf{m} \beta}\right)\right] \text { and } \\
& \hat{\Phi}_{\alpha \lambda}(\mathbf{k})=\frac{1}{\sqrt{N}} \sum_{\mathbf{n}} \hat{\Phi}_{\mathbf{n} \alpha \lambda} \exp \left(-i \mathbf{k} \cdot \mathbf{r}_{\mathbf{n} \alpha}\right)
\end{aligned}
$$

Here $N$ is the number of elementary cells constituting the lattice. Notice that the wave vector $\mathbf{k}$, which characterizes eigenstates of

*Corresponding author: Rumyantsev V, A.A. Galkin Donetsk Institute for Physics and Engineering, Donetsk, Ukraine, Tel: (062) 311-52-27; E-mail: 380957931135@yandex.ru

Received August 20, 2017; Accepted August 28, 2017; Published September 20, 2017

Citation: Rumyantsev V, Aparajita U, Roslyak O (2017) Effect of Elastic Deformation on the Dispersion Characteristics of a Chain of Microcavities. J Laser Opt Photonics 4: 165. doi: 10.4172/2469-410X.1000165

Copyright: (C) 2017 Rumyantsev V, et al. This is an open-access article distributed under the terms of the Creative Commons Attribution License, which permits unrestricted use, distribution, and reproduction in any medium, provided the original author and source are credited. 
electromagnetic excitations, varies within the first Brillouin zone. The zone itself is a function of a uniform deformation $\hat{\varepsilon}$.

Generally speaking eigenvalues of Hamiltonian (1) may be found via Bogolyubov-Tyablikov transformation [9]. This yields the following equation for elementary excitation spectrum $\Omega(\mathbf{k}, \hat{\varepsilon})$ :

$$
\operatorname{det}\left\|D_{\alpha \beta}^{\lambda \sigma}(\mathbf{k}, \hat{\varepsilon})-\hbar \Omega(\mathbf{k}, \hat{\varepsilon}) \delta_{\alpha \beta} \delta_{\lambda \sigma}\right\|=0
$$

Below we carry out a detailed investigation of the spectrum $\hbar \Omega(\mathbf{k}, \hat{\varepsilon})$, which holds valuable information about the effective mass of such collective excitations and some other quantities of interest.

\section{Results and Discussion}

\section{Exciton-like excitations in a one-dimensional microcavity lattice under a uniform elastic deformation}

To be more specific, let us assume that under a uniform deformation described by tensor $\hat{\varepsilon}$ each cavity changes its position in such fashion that the lattice constant $d(\varepsilon)$ has the form:

$$
d(\varepsilon)=(1+\varepsilon) d_{0},
$$

here $d_{0}$ corresponds to a strain-free structure, and $\varepsilon$ is the corresponding component of the stress tensor directed along the chain. The reciprocal lattice constant $b(\varepsilon)$ is found from the standard relation:

$$
b(\varepsilon) \cdot d(\varepsilon)=2 \pi
$$

In what follows, we assume that the microcavity array is made up of two sublattices void of quantum emitters. Position of microcavities is defined by the equality $r_{n \alpha}(\varepsilon)=r_{n}(\varepsilon)+r_{\alpha}(\varepsilon)$, and therefore their positions in the zeroth cell of the first and second sublattice $\left(r_{n=0}=0\right)$ are correspondingly: $r_{01}=0$ and $r_{02}(\varepsilon)=a(\varepsilon)$. The spectrum of excitonlike excitations $\Omega(k, \varepsilon)$ is found from relation (4):

$$
\left\|\begin{array}{cc}
\hbar \Omega(k, \varepsilon)-\hbar \omega_{1}^{p h} & A_{12}(k, \varepsilon) \\
A_{21}(k, \varepsilon) & \hbar \Omega(k, \varepsilon)-\hbar \omega_{2}^{p h}(\varepsilon)
\end{array}\right\|=0 .
$$

Quantities $A_{\alpha \beta}(k, \varepsilon)$ in Eq. (7) are Fouriertransforms of matrix $A_{n \alpha m \beta}(\varepsilon)$ of resonance interaction: $A_{\alpha \beta}(k, \varepsilon)=\sum_{m} A_{n \alpha m \beta}(\varepsilon) \exp \left\{i k\left[r_{n \alpha}(\varepsilon)-r_{m \beta}(\varepsilon)\right]\right\}$. In the frame of our model within the nearest-neighbor approximation, the matrix elements $A_{\alpha \beta}(k, \varepsilon)$ take the following form:

$$
\begin{aligned}
& A_{12}(k, \varepsilon) \cong A_{12}[a(\varepsilon)] \exp [-i k \cdot a(\varepsilon)]+A_{12}[d(\varepsilon)-a(\varepsilon)] \exp \{-i k \cdot[d(\varepsilon)-a(\varepsilon)]\}, \\
& A_{21}(k, \hat{\varepsilon})=A_{21}[a(\varepsilon)] \exp [i k \cdot a(\varepsilon)]+A_{21}[d(\varepsilon)-a(\varepsilon)] \exp \{i k \cdot[d(\varepsilon)-a(\varepsilon)]\}
\end{aligned}
$$

According to Rumyantsev et al. [6], quantities $A_{12(21)}[a(\varepsilon)]$, which are components of the matrix $A_{n \alpha m \beta}(\varepsilon)$ describing the resonance interactions, correspond to nearest neighbors equal to $A_{12(21)}[a(\varepsilon)]=A_{12(21)}(a) \exp (-\varepsilon), \quad A_{12(21)}[d(\varepsilon)-a(\varepsilon)]=A_{12(21)}(d-a) \exp (-\varepsilon) . \quad$ In our case we put $A_{12}(a) \simeq A_{21}(a), A_{12}(d-a) \simeq A_{21}(d-a)$. Relation

(4) shows that the dispersion law $\Omega(k, \varepsilon)$ of elementary electromagnetic excitations is determined both by frequency characteristics of resonator array and by the explicit form of $A(k, \varepsilon)$, as well as by the nature of deformation (e.g., by a uniaxial extension $\varepsilon>0$ or contraction $\varepsilon<0$ ).

Further calculations are performed for a uniaxial deformation of a uniform isotropic one-dimensional medium. The following modeling parameters are adopted: frequencies of resonance photonic modes in cavities (independent of deformation $(\varepsilon)$ are $\omega_{1}^{p h}=2 \pi \times 311 \mathrm{THz} \quad$ and $\omega_{2}^{p h}=2 \pi \times 331 \mathrm{THz} \quad$ and $A_{12}(a) / 2 \hbar=3.5 \cdot 10^{14} \mathrm{~Hz}$, $A_{12}(d-a) / 2 \hbar=1.2 \cdot 10^{14} \mathrm{~Hz}, a=1 \cdot 10^{-7} \mathrm{~m}, \quad d=9 \cdot 10^{-7} \mathrm{~m}$. Figure 1 depicts the dependence $\Omega_{v}(k, \varepsilon),(v=1,2)$, of the deformed one-dimensional lattice for different values of $\varepsilon$. Shaded region in the $(k, \varepsilon)$-plane corresponds to the first Brillouin zone.

An important property of the band gap photonic structures is their ability to produce the so-called "slow light". It has important application for designing quantum optical information processing devices. The effective decrease of quasiparticle group velocity was shown to occur in coupled wave-guide optical resonators [10] and in various types of multilayer semiconductor structures [11]. A key role in decreasing the group velocity is played by the character of quasi particles' effective mass $m_{\text {eff }(v)}(v=1 ; 2)$. Figure 2 depicts the dependence of the effective mass $m_{\text {eff }(v)}=\hbar\left[\left.\frac{\partial^{2} \Omega_{v}(k, \varepsilon)}{\partial k^{2}}\right|_{k=0}\right]^{-}$of exciton-like excitations on the degree of deformation. Its examination indicates that a careful choice of $\varepsilon$ permits to attain the necessary parameters of the "slow light".

\section{Conclusion}

The study of the spectrum of elementary electromagnetic excitations in a one-dimensional array of tunnel-coupled microcavities shows

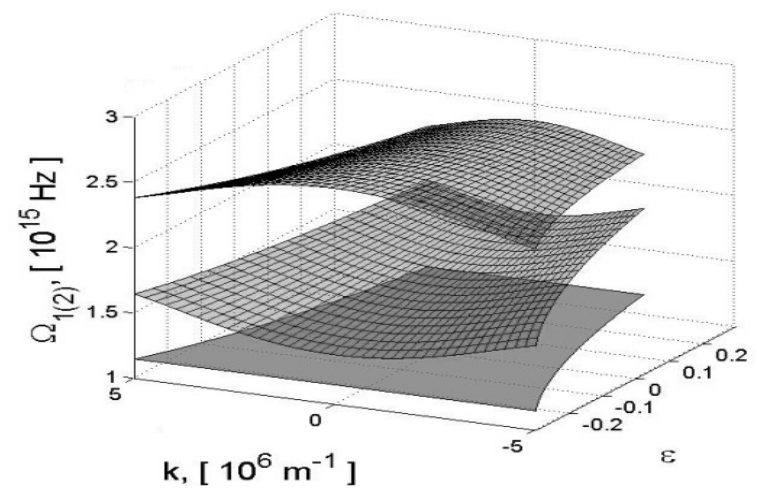

Figure 1: Dependencies $\Omega_{1(2)}(k, \varepsilon)$ of the deformed one-dimensional lattice.

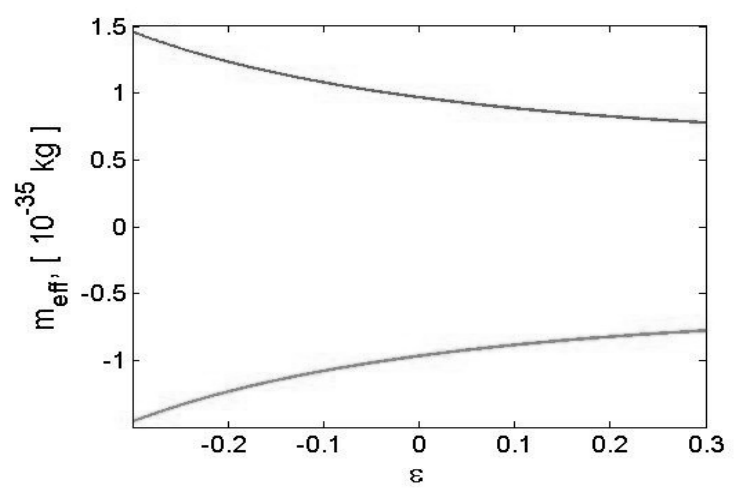

Figure 2: Dependence of the effective mass of exciton-like excitations on deformation degree. 
Citation: Rumyantsev V, Aparajita U, Roslyak O (2017) Effect of Elastic Deformation on the Dispersion Characteristics of a Chain of Microcavities. J Laser Opt Photonics 4: 165. doi: 10.4172/2469-410X.1000165

Page 3 of 3

that subjecting the system to controlled elastic stress is an effective tool for altering its energy structure and optical properties. We have demonstrated that the conclusion holds for the cases of microresonator arrays with embedded quantum dots as well as for quantum-dot-free lattices. The presence of deformation and structural defects may lead to the increase of the effective mass of corresponding excitations and therefore to a decrease of their group velocity. The results of numerical simulations performed on the basis of the constructed model contribute to modeling of the new class of functional materials photonic crystalline system constituted of coupled microcavities. Their capabilities include the controllable propagation of electromagnetic excitations.

\section{References}

1. Sedov ES, Alodjants AP, Arakelian SM, Lin YY, Lee RK (2011) Nonlinear properties and stabilities of polaritonic crystals beyond the low-excitationdensity limit. Phys Rev A 84: 013813.

2. Vahala KJ (2003) Optical microcavities. Nature 424: 839.

3. Kaliteevskii MA (1997) Coupled vertical microcavities. Tech Phys Lett 23: 120.
4. Golubev VG, Dukin AA, Medvedev AV, Pevtsov AB, Sel'kin AV, et al (2003) Splitting of resonant optical modes in Fabry-Perot microcavities. Semiconductors 37: 832

5. Rumyantsev VV, Fedorov SA, Gumennyk KV, Sychanova MV, Kavokin AV (2014) Exciton-like electromagnetic excitations in non-ideal microcavity supercrystals. Nature Sci Rep 4: 6945.

6. Rumyantsev VV, Fedorov SA, Gumennyk KV (2016) Polaritons in a nonideal array of ultracold quantum dots. Low Temperature Physics 42: 447-454.

7. Rumyantsev V, Fedorov S, Sychanova M (2015) Peculiarities of light-matter coupling in imperfect lattice of coupled microresonators. J Laser Opt Photonics 2: 113.

8. Joannopoulos JD, Johnson SG, Winn JN, Meade RD (2008) Photonic Crystals Molding the Flow of Light, (2nd edn)., Princeton: Princeton University Press.

9. Agranovich VM (1968) Theory of Excitons. Moscow. Nauka Publishers.

10. Yang ZS, Kwong NH, Binder R, Smirl AL (2005) Stopping, storing, and releasing light in quantum-well Bragg structures. J Opt Soc Am VB 22: 2144-2156.

11. Turukhin AV, Sudarshanam VS, Shahriar MS, Musser JA, Ham BS, et al. (2002) Phys Rev Lett V 88: 023602-1. 